\title{
Mutual impact of clinically translatable near-infrared dyes on photoacoustic image contrast and in vitro photodynamic therapy efficacy (Erratum)
}

\author{
Ljubica Z. Petrovic, ${ }^{\dagger}$ Marvin Xavierselvan, ${ }^{\dagger}$ Maju Kuriakose, ${ }^{\dagger}$ \\ Michael D. Kennedy, Christopher D. Nguyen, Julian J. Batt, \\ Kelsey B. Detels, and Srivalleesha Mallidi* \\ Tufts University, Department of Biomedical Engineering, Medford, Massachusetts, \\ United States
}

[DOI: 10.1117/1.JBO.25.6.069802]

This article [J. Biomed. Opt. 25(06), 0638008 (2020) doi: 10.1117/1.JBO.25.6.063808] was originally published online on 28 February 2020 with mislabeled $y$-axes in Fig. 4 and Fig. S2, panels (a)-(c). The correct label is "Absorbance (\%)." The data in the figures remains the same.

This article was corrected online on 25 June 2020.

*Address all correspondence to Srivalleesha Mallidi, E-mail: srivalleesha.mallidi@tufts.edu

${ }^{\dagger}$ Equal contribution. 